УДК 539.3

\title{
Rotation versus curvature fractal scaling in bending failure
}

\author{
Alberto Carpinteri and Federico Accornero \\ Department of Structural, Geotechnical and Building Engineering, Politecnico di Torino, Torino, 10129, Italy
}

In the present work, an approach based on fractal geometry, that has been successfully applied for the tensile and the compressive behaviour of quasi-brittle materials, is followed in order to obtain a fractal scaling from three-point bending tests of concrete specimens. According to this approach, bending strength, fracture energy, and the critical kinematic parameter, represented by the localized rotation, are not defined with respect to canonical physical dimensions, but on fractal sets presenting noninteger dimensions. In the case of bending strength, the dimensional decrement of the reference area $d_{\sigma}$ represents the weakening of the reacting cross section, whereas in the case of fracture energy the dimensional increment $d_{G}$ represents the roughness of the fracture surface. Furthermore, the kinematic parameter proves to be intermediate between a dimensionless rotation angle and a curvature, i.e., it moves from a generalized displacement to a deformation: its dimensional decrement $d_{\chi}$ represents the curvature localization along the beam span according to a lacunar fractal constituted by the infinite radial cracks converging to the center of curvature. As a consequence, the classical mechanical parameters are replaced by fractal quantities, which represent the actual material properties.

Keywords: fractal scaling, bending strength, fracture energy, rotation, curvature

DOI $10.24411 / 1683-805 X-2018-16008$

\section{Фрактальный скейлинг зависимости угла поворота от кривизны при изгибном разрушении}

\author{
A. Carpinteri, F. Accornero
}

Туринский политехнический университет, Турин, 10129, Италия

\begin{abstract}
В работе изучен фрактальный скейлинг при испытаниях бетонных образцов на трехточечный изгиб с использованием подхода на основе фрактальной геометрии, который был успешно применен для описания поведения квазихрупких материалов в условиях растяжения и сжатия. В рамках данного подхода изгибная прочность, энергия разрушения и критический кинематический параметр, представленный локализованным вращением, определяются не по каноническим физическим размерностям, а по фрактальным множествам, представляющим нецелые размерности. В случае прочности на изгиб размерный декремент условной площади $d_{\sigma}$ представляет собой ослабление активного поперечного сечения, тогда как в случае энергии разрушения размерным инкрементом $d_{G}$ является шероховатость поверхности разрушения. Кинематический параметр находится в промежутке между безразмерным углом поворота и кривизной, т.е. переходит от обобщенного смещения к деформации: его размерный декремент $d_{\chi}$ представляет локализацию кривизны вдоль луча в соответствии с лакунарным фракталом, который образован бесконечными радиальными трещинами, сходящимися к центру кривизны. В результате классические механические параметры заменяются фрактальными величинами, которые представляют фактические свойства материала.
\end{abstract}

Ключевые слова: фрактальный скейлинг, прочность на изгиб, энергия разрушения, вращение, кривизна

\section{Introduction: Fractal scaling of bending strength, fracture energy, and critical rotation angle}

Progress in understanding complex physical processes is closely connected with the recognition of geometrical patterns in experimental evidences and computational results. In the framework of dimensional analysis, a physical process can be reduced in order to determine a function of a single dimensionless variable: it is the case described by the material scale length for plastic materials [1], or the brittleness number for aggregative materials $[2,3]$. The correct choice of the set of governing parameters is not only the result of the application of a formal procedure, but requires good intuition and a great deal of attention to the qualitative analysis of the phenomenon under consideration 
[4]. This preliminary identification of forms and the search for invariant relations constitute the foundation of the intermediate-asymptotic representation of a wide range of physical problems, mainly governed by scaling or powerlaw relationships [5, 6]. They give evidence of a very deep property of the phenomena under consideration: their selfsimilarity [7].

Indeed self-similar solutions are always "intermediate asymptotics" to the solutions of general problems valid for times and distances from boundaries large enough for the influence of the fine details to disappear, but small enough that the system is far from the ultimate equilibrium state [5, 8-10].

A very powerful tool to study critical phenomena, which exploits self-similarity, is the renormalization group theory [11]. It analyzes the universal properties of systems displaying scale invariance, as typically occurs at critical points. It was successfully applied to electromagnetism [12] and, later on, to solid mechanics $[13,14]$.

Self-similarity is also the main property of fractal sets, whose basic features and properties can effectively model the microstructure of heterogeneous quasi-brittle materials [13-17].

In the framework of fracture mechanics and material science, the cohesive crack model $[18,19]$ is one of the most widespread models when dealing with quasi-brittle materials. Generally speaking, the cohesive law is regarded as a material property. Nevertheless, experimental tests have shown that the cohesive law parameters depend on the structural size: the material strength decreases with increasing sizes, whereas the fracture energy increases. This dependence is usually referred to as size effect $[2,3]$. Although such an effect is evident only when a sufficiently broad range of sizes is considered, the size effect is not a mere speculation, since it is a common procedure to extrapolate from the laboratory tests, i.e. small sized specimens, the cohesive law parameters that are then used to simulate the structural behaviour of real structures, i.e. large sized specimens. The importance of correctly addressing the size effect on material strength, fracture energy and critical kinematic parameter is therefore evident.

Accordingly, the size effects on the cohesive crack model parameters are described by power-laws, that are deeply investigated in $[13,14]$, where the size-independent or fractal cohesive crack model is introduced. As a matter of fact, fractal geometry highlighted that self-similar objects

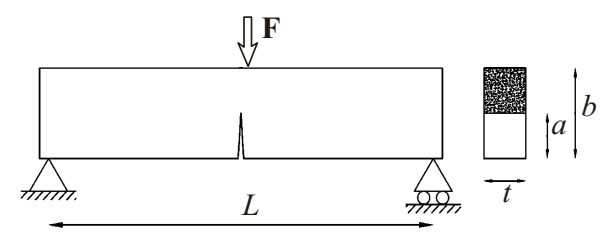

Fig. 1. Prenotched plain concrete beam specimen

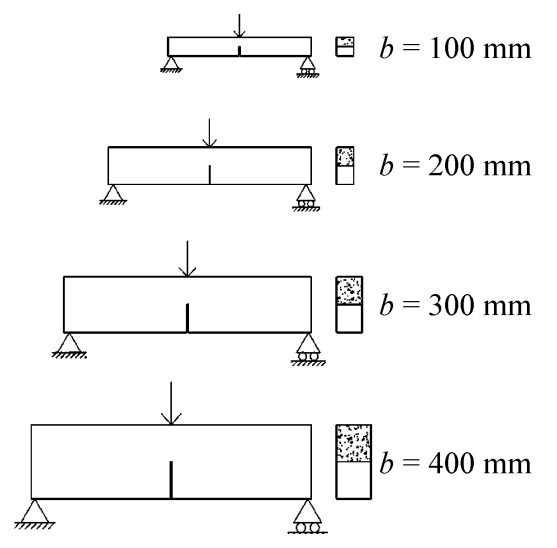

Fig. 2. Specimen sizes

can be characterized by noninteger geometrical dimensions. Both renormalization and fractals imply the same scaling of a power-law type.

Basically, the fractal view of material science requires the introduction of new mechanical quantities: the fractal stress, the fractal strain, and the fractal dissipated energy. Scientists and engineers are used to deal with quantities with integer physical dimensions, although an effective, size-independent material description may be achieved only with noninteger physical quantities [20-22].

In the present work, the approach based on fractal geometry, that has been successfully applied for the tensile and the compressive behaviour of quasi-brittle materials $[23,24]$, is followed in order to obtain a fractal scaling from bending tests of concrete beams. According to this approach, bending strength, fracture energy, and critical localized rotation are not defined with respect to canonical physical dimensions, but on fractal sets presenting noninteger dimensions. In particular, the kinematic parameter proves to be intermediate between a dimensionless rotation angle and a curvature, i.e., it moves from a generalized displacement to a deformation. As a consequence of this fractal interpretation and transition, the abovementioned classical mechanical parameters should be replaced by fractal quantities, which represent the actual material properties.

\section{Outline of the experiments}

In order to study scale effects on bending strength and fracture energy, a set of three-point bending (TPB) tests is

\begin{tabular}{c|c|c}
\hline \multicolumn{2}{c}{} & Table \\
\hline $100 \pm 5$ & $t, \mathrm{~mm}$ & $L, \mathrm{~mm}$ \\
\hline $200 \pm 5$ & $100 \pm 5$ & $800 \pm 5$ \\
\hline $300 \pm 5$ & $100 \pm 5$ & $1130 \pm 5$ \\
\hline $400 \pm 5$ & $150 \pm 5$ & $1385 \pm 5$ \\
\hline
\end{tabular}




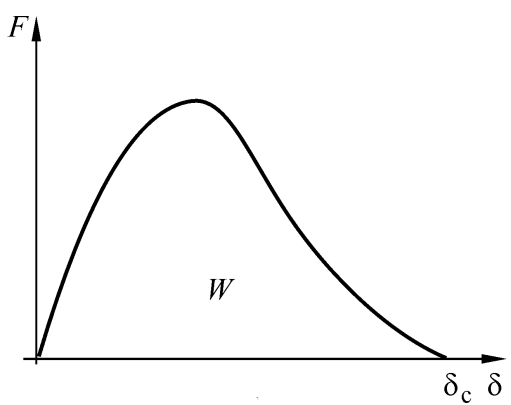

Fig. 3. Load versus deflection curve [25]

performed on prenotched plain concrete specimens (Fig. 1). These tests are conducted following RILEM recommendations [25], and considering the notch depth $a$, equal to the beam half-depth $b / 2$ (table). TPB tests are performed exploiting a servohydraulic MTS universal testing machine. All the specimens are tested until final failure.

The tests involving the specimens with $b=100 \mathrm{~mm}$ are controlled by the crack mouth opening displacement (CMOD), while the other ones are conducted by the vertical displacement $\delta$ of the hydraulic jack (deflection). In all cases, a stable post-peak behaviour is obtained.

Figure 2 shows the specimen sizes recommended by RILEM Technical Committe (table).

The result of each test is a curve representing the load $F$, as a function of the deflection $\delta$, as displayed in Fig. 3 . The area under this diagram gives the energy $W$, supplied by the machine [25].

\section{Experimental results: Load-deflection curves}

Figure 4 shows the superimposed load-deflection curves obtained from the TPB tests of three plain concrete specimens with $b=100 \mathrm{~mm}$, two specimens with $b=200 \mathrm{~mm}$, one specimen with $b=300 \mathrm{~mm}$, and three specimens with $b=400 \mathrm{~mm}$.

For each test, it is possible to evaluate fracture energy and bending strength. These values are interpreted in the following by fractal scaling laws.

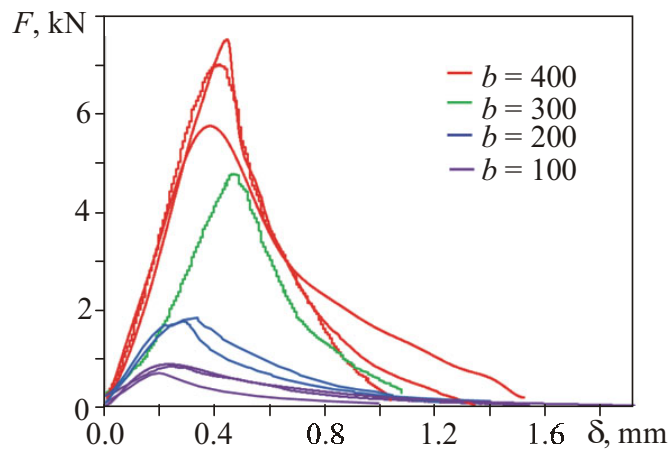

Fig. 4. Experimental load-deflection curves from TPB tests (color online)

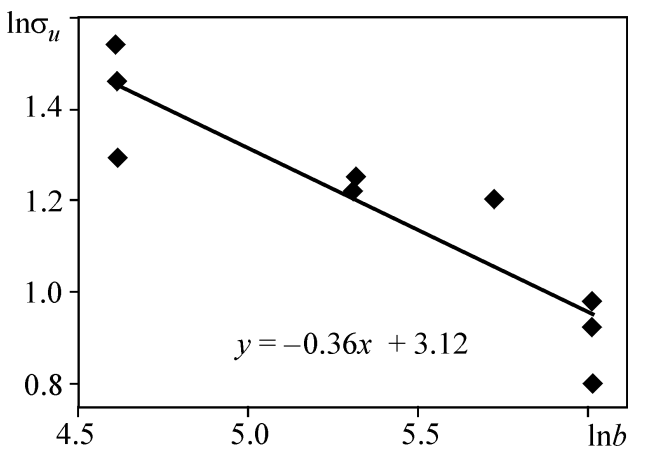

Fig. 5. Size effect on apparent bending strength

\section{Scale effects on bending strength}

The values of apparent bending strength obtained from TPB tests can be graphically represented in a bilogarithmic diagram (Fig. 5). A fractal scaling law is used to fit the experimental results, providing the value of the renormalized scale-independent bending strength $\sigma_{u}^{*}$ and the fractal exponent $d_{\sigma}[13,14]$ :

$$
\sigma_{u}=\sigma_{u}^{*} b^{-d_{\sigma}} \text {. }
$$

Hence, the weakening of the ligament is characterised by the dimensional decrement with respect to an area, $d_{\sigma}=$ $=0.36$, which provides a fractal domain of dimension equal to 1.64 .

For each test, the value of $\ln \sigma_{u}^{*}$ can be represented as in Fig. 6, deducing the renormalized bending strength, $\sigma_{u}^{*}=22.6 \mathrm{~N} \mathrm{~mm}^{-1.64}$.

\section{Scale effects on fracture energy}

A power-law can be used for highlighting the scale effect on fracture energy $[13,14]$ :

$$
G_{\mathrm{f}}=G_{\mathrm{f}}^{*} b^{d_{G}} \text {. }
$$

The experimental results concerning this apparent characteristic are represented in a bilogarithmic diagram (Fig. 7), which is due to the roughness of the fracture surface: it involves the dimensional increment $d_{G}=0.19$. This means that the energy dissipation occurs in a fractal domain with dimension 2.19 .

The values of $\ln G_{\mathrm{f}}^{*}$ are represented for all the tests in Fig. 8. It shows the renormalized scale-independent value of the fracture energy $G_{\mathrm{f}}^{*}=209.9 \mathrm{Jm}^{-2.19}=209.9 \mathrm{Nm}^{-1.19}$.

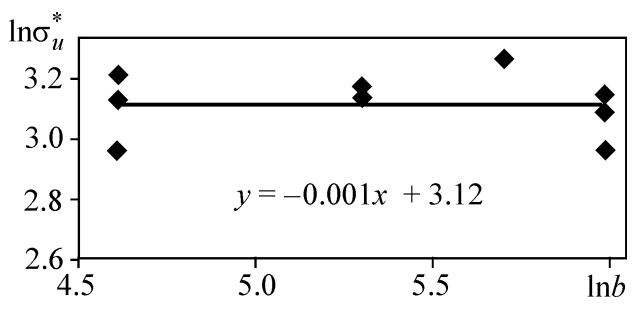

Fig. 6. Renormalized bending strength 


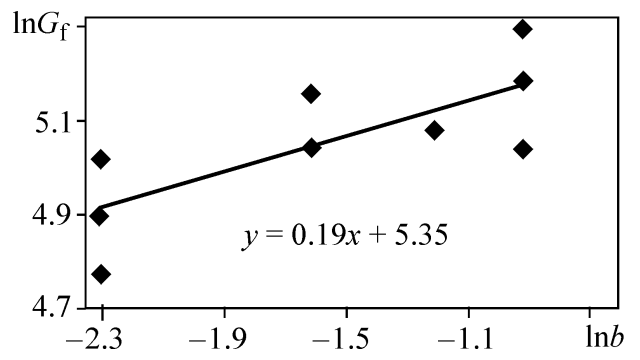

Fig. 7. Size effect on apparent fracture energy

Renormalized bending strength and fracture energy represent the target of the renormalization procedure, i.e., the determination of physical quantities that are invariant under a change of observation scale $[13,14]$ : this means that, for size scales where random self-similarities hold, the universal mechanical properties $\sigma_{u}^{*}$ and $G_{\mathrm{f}}^{*}$ are constant, although they are represented by unusual physical dimensions.

\section{Scale effects on the kinematic parameter}

It was observed considering the critical displacement $w_{\mathrm{c}}$ in uniaxial tensile tests [20], that the correspondent fractal kinematic quantity can be represented by the renormalized axial strain $\varepsilon_{\mathrm{c}}^{*}$. Nevertheless, in the case of TPB tests, it may be difficult to take into consideration the axial strain, because it varies along the depth of the cross section. Moreover, also the curvature $\chi$ may be not immediately useful to describe the damage process, because it presents a discontinuity in correspondence of the cracked cross section.

For these reasons, the most suitable kinematic parameter, able to describe the critical point in TPB tests, is the localized rotation at the beam midspan. In order to evaluate the critical value of this parameter, i.e., the rotation angle at failure, some geometric considerations are made, based on Fig. 9.

At failure, the rotation angle can be evaluated as

$$
\varphi_{\mathrm{c}}=\frac{\delta_{\mathrm{c}}}{L / 2} \text {. }
$$

It is possible to define the critical value of the localized rotation for each TPB test: these values are graphically represented in the bilogarithmic diagram of Fig. 10.

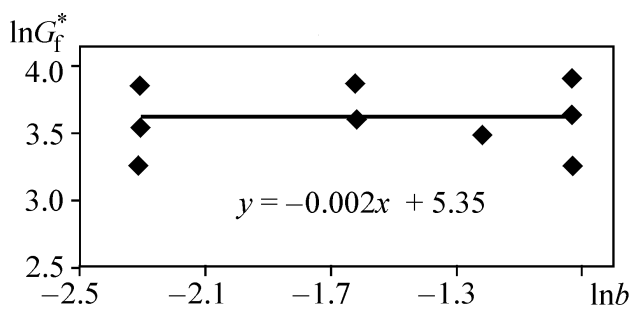

Fig. 8. Renormalized fracture energy

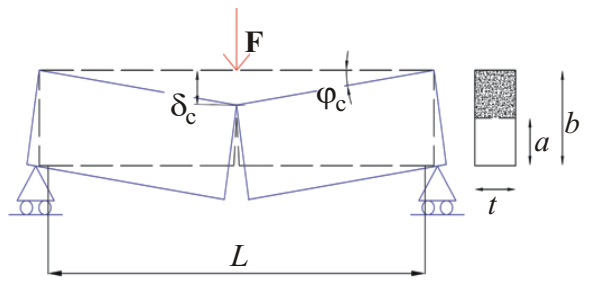

Fig. 9. Localized rotation at the beam midspan (color online)

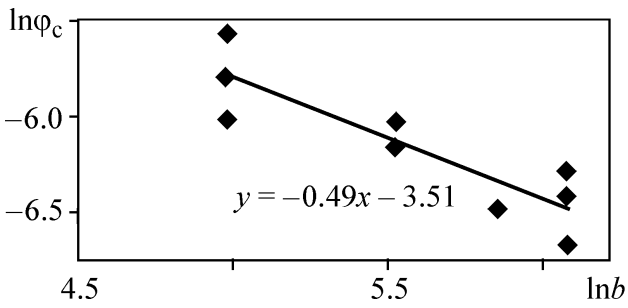

Fig. 10. Size effect on critical rotation angle

A power-law can be proposed to describe the scale effects on the critical value of the kinematic parameter for TPB tests:

$$
\varphi_{\mathrm{c}}=\varphi_{\mathrm{c}}^{*} b^{-d_{\chi}},
$$

where $d_{\chi}=0.49$. Hence, from Eq. (4) the renormalized critical rotation $\varphi_{c}^{*}$ results to be intermediate between a dimensionless rotation angle and a radius of curvature. The inverse of this parameter returns the renormalized critical curvature $\chi_{\mathrm{c}}^{*}$.

The values of $\ln \chi_{c}^{*}$ can be represented in the bilogarithmic diagram of Fig. 11, deducing the renormalized critical curvature, $\chi_{c}^{*}=33.45 \mathrm{~mm}^{-0.49}$.

As is shown, the kinematic parameter proves to be intermediate between a dimensionless rotation angle and a curvature, i.e., it moves from a generalized displacement to a deformation. The dimensional decrement $d_{\chi}$ represents the curvature localization along the beam spam according to a lacunar fractal constituted by the infinite radial cracks converging to the center of curvature.

The remarkable dispersion between the different curves in Fig. 2 due to the scale effects can be minimized by introducing a correct renormalization of the load-deflection dia-

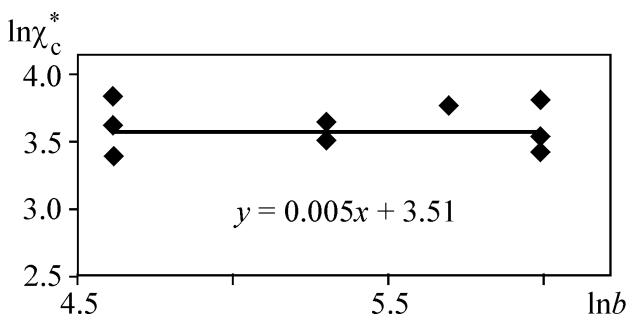

Fig. 11. Renormalized critical curvature 


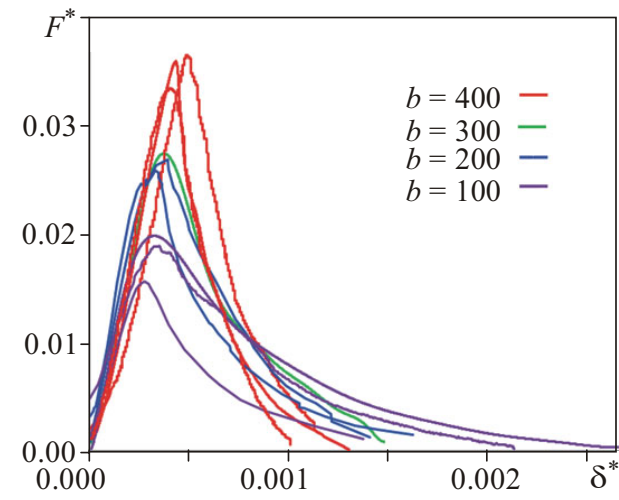

Fig. 12. Renormalized load-deflection curves from TPB tests (color online)

grams. In order to obtain a decrease in dispersion, it is necessary to use the renormalized bending strength $\sigma_{u}^{*}$ and the rescaled dimension of the ligament. In particular, the dimensionless value of the load turns into

$$
F^{*}=\frac{F}{\sigma_{u}^{*} t b^{1-d_{\sigma}}} .
$$

In the same way, the dimensionless value of the deflection can be evaluated as

$$
\delta^{*}=\frac{\delta \chi_{c}^{*}}{b^{1-d_{\chi}}}
$$

The renormalized load-deflection curves are represented as overlapping in Fig. 12.

Thus, considering the fracture energy definition as proposed by RILEM [25], and the renormalized critical values $\sigma_{u}^{*}$ and $\chi_{\mathrm{c}}^{*}$, we have

$$
\begin{aligned}
G_{\mathrm{f}}^{*} & =\frac{\sigma_{u}^{*} \int_{\mathrm{c}}^{*}}{\chi_{\mathrm{c}}^{*}} F_{0}^{*} \mathrm{~d} \delta^{*}=\frac{1}{b^{1-d_{\sigma}-d_{\chi}}} \frac{1}{t b} \int_{0}^{\delta_{\mathrm{c}}} F \mathrm{~d} \delta= \\
& =\frac{1}{b^{1-d_{\sigma}-d_{\chi}}} G_{\mathrm{f}} .
\end{aligned}
$$

Recalling Eq. (2), the fundamental relation between the three scaling exponents $[23,24]$ is found again

$$
d_{\sigma}+d_{\chi}+d_{G}=1 \text {. }
$$

The experimental validation of Eq. (8) is then provided:

$$
d_{\sigma}+d_{\chi}+d_{G}=0.36+0.49+0.19=1.04 \cong 1 \text {. }
$$

\section{Conclusions}

Fractal models of material strength and fracture energy indicate the need for a significant change in the framework of measuring material properties in solid mechanics. When dealing with disordered materials with fractal microstructure, the concept of ideal reference areas for material ligaments and fracture surfaces must be dramatically revised. Moreover, fractal geometry permits to give a unified explanation to the size effects on mechanical properties of quasi-brittle materials, otherwise impossible to obtain. In the present work, a fractal scaling is obtained from three- point bending tests of concrete specimens. Bending strength, fracture energy, and localized rotation are not defined with respect to canonical physical dimensions, but on fractal sets presenting noninteger dimensions. In the case of bending strength, the dimensional decrement of the reference area $d_{\sigma}$ represents the weakening of the reacting cross section, whereas in the case of fracture energy the dimensional increment $d_{G}$ represents the roughness of the fracture surface. Furthermore, the dimensional decrement $d_{\chi}$ represents the curvature localization along the beam span according to a lacunar fractal constituted by the infinite radial cracks converging to the center of curvature. In this way, the classical mechanical parameters are replaced by fractal quantities, which represent the actual material properties. Finally, an experimental validation of the fundamental relationship among the scaling exponents of bending strength, fracture energy, and critical rotation angle is provided.

\section{References}

1. Goldstein R.V., Vainshelbaum V.M. Material scale length as a measure of fracture toughness in fracture mechanics of plastic materials // Int. J. Fract. - 1978. - V. 14. - P. 185-201.

2. Carpinteri A. Size Effect in Fracture Toughness Testing: A Dimensional Analysis Approach // Proc. Int. Conf. Analytical and Experimental Fracture Mechanics, Roma, Italy // Ed. by G.C. Sih, M. Mirabile. - Alphen an den Rijn: Sijthoff \& Noordhoff, 1980. - P. 785 797.

3. Carpinteri $A$. Notch sensitivity in fracture testing of aggregative materials // Eng. Fract. Mech. - 1982. - V. 16. - P. 467-481.

4. Barenblatt G.I. Flow, Deformation and Fracture. - Cambridge: Cambridge University Press, 2014.

5. Barenblatt G.I. Similarity, Self-Similarity, and Intermediate Asymptotics. - New York: Springer, 1979.

6. Barenblatt G.I. Scaling, Self-Similarity, and Intermediate Asymptotics. - Cambridge: Cambridge University Press, 1996.

7. Zeldovich Y.B., Barenblatt G.I. On the asymptotic properties of selfsimilar solutions of the equations of unsteady gas filtration // Dokl. USSR Acad. Sci. - 1958. - V. 118. - P. 671-674.

8. Barenblatt G.I., Botvina L.R. Incomplete self-similarity of fatigue in the linear range of crack growth // Fatig. Fract. Eng. Mater. Struct. 1980. - V. 3. - P. 193-202.

9. Barenblatt G.I., Botvina L.R. A note concerning power-type constitutive equations of deformation and fracture of solids // Int. J. Eng. Sci. - 1982. - V. 20. - P. 187-191.

10. Barenblatt G.I., Botvina L.R. Self-similar nature of fatigue failure: damage accumulation // Mech. Solid. - 1983. - V. 18. - P. 160-164.

11. Wilson K.G. Renormalization group and critical phenomena// Phys. Rev. B. - 1971. - V. 4. - P. 3174-3183.

12. Wilson K.G., Kogut J. The renormalization group and the $\varepsilon$ expansion // Phys. Rep. - 1974. - V. 12. - P. 75-199.

13. Carpinteri $A$. Fractal nature of material microstructure and size effects on apparent mechanical properties // Mech. Mater. - 1994. V. 18. - P. 89-101; (1992) Internal Reports, Laboratory of Fracture Mechanics, Politecnico di Torino, 1/92.

14. Carpinteri A. Scaling laws and renormalization groups for strength and toughness of disordered materials // Int. J. Solid. Struct. - 1994. V. 31. - P. 291-302.

15. Goldstein R.V., Mosolov A.B. Fractal cracks // J. Appl. Math. Mech. 1992. - V. 56. - P. 563-571.

16. Mandelbrot B.B. The Fractal Geometry of Nature. - San Francisco: Freeman, 1982. 
17. Turcotte D.L. Fractals and Chaos in Geology and Geophysics. - Cambridge: Cambridge University Press, 1992.

18. Barenblatt G.I. The mathematical theory of equilibrium cracks in brittle fracture // Adv. Appl. Mech. - 1962. - V. 7. - P. 55-129.

19. Carpinteri A. Cusp catastrophe interpretation of fracture instability // J. Mech. Phys. Solids. - 1989. - V. 37. - P. 567-582.

20. Carpinteri A., Ferro G. Size effects on tensile fracture properties: A unified explanation based on disorder and fractality of concrete microstructure // Mater. Struct. - 1994. - V. 27. - P. 563-571.

21. Carpinteri A., Ferro G. Scaling behaviour and dual renormalization of experimental tensile softening responses // Mater. Struct. - 1998. V. 31. - P. 303-309.
22. Carpinteri A., Chiaia B. Multifractal scaling laws in the breaking behaviour of disordered materials // Chaos, Solitons Fractals. - 1997. V. 8. - P. $135-150$.

23. Carpinteri A., Chiaia B., Cornetti P. A scale-invariant cohesive crack model for quasi-brittle materials // Eng. Fract. Mech. - 2002. - V. 69. P. 207-217.

24. Carpinteri A., Corrado M. An extended (fractal) overlapping crack model to describe crushing size-scale effects in compression // J. Eng. Fail. Anal. - 2009. - V. 16. - P. 2530-2540.

25. RILEM Technical Committee 50-FMC. Determination of the fracture energy of mortar and concrete by means of three-point bend tests on notched beams // Mater. Struct. - 1985. - V. 18. - P. 287-290.

Received November 15, 2018, revised November 15, 2018, accepted November 22, 2018

Сведения об авторах

Alberto Carpinteri, Full Professor, Politecnico di Torino, Italy, alberto.carpinteri@polito.it

Federico Accornero, Research Fellow, Politecnico di Torino, Italy, federico.accornero@polito.it 